\title{
BLIND DECONVOLUTION USING A REGULARIZED STRUCTURED TOTAL LEAST NORM ALGORITHM *
}

\author{
ARMIN PRUESSNER ${ }^{\dagger}$ AND DIANNE P. O’LEARY ${ }^{\ddagger}$
}

\begin{abstract}
Rosen, Park and Glick proposed the structured total least norm (STLN) algorithm for solving problems in which both the matrix and the right-hand side contain errors. We extend this algorithm for ill-posed problems by adding regularization and use the resulting algorithm to solve blind deconvolution problems as encountered in image deblurring when both the image and the blurring function have uncertainty. The resulting regularized structured total least norm (RSTLN) algorithm preserves any affine structure of the matrix and minimizes the discrete $L_{p}$-norm error, where $p=1,2$, or $\infty$. We demonstrate the effectiveness of these algorithms for blind deconvolution.
\end{abstract}

Key words. least squares, total least squares, total least norm, structured total least norm, minimization, regularization, ill-posed problem, 1-norm, 2-norm, $\infty$-norm, overdetermined linear system, blind deconvolution, image deblurring, boundary conditions, constrained total least squares

AMS subject classifications. 65F22, 65K10, 90C05

1. Introduction and Background. Most image recording devices fail to record the intensity of a given image scene exactly. Each recorded image section (or pixel) describing the corresponding scene has errors in the form of either random noise, blurring, or both. Blurring occurs when the recorded intensity of a given pixel is in effect influenced by the intensity of neighboring sections. Because of these imperfections in recorded images, it is often necessary to apply deblurring techniques to obtain clearer images.

The problem of image deblurring is modeled as an integral equation of the first kind:

$$
\int_{\Omega} a(s, t) x(t) d t=b(s)-\mu(s)=b_{c}(s)
$$

where $s, t \in \mathbf{R}^{2}$ are the spatial coordinates, $\Omega$ the domain or (nonzero) support of the image, $x: \mathbf{R}^{2} \rightarrow \mathbf{R}$ the true image, $a: \mathbf{R}^{4} \rightarrow \mathbf{R}$ the point spread function, and $\mu: \mathbf{R}^{2} \rightarrow \mathbf{R}$ random noise. The function $b(s)$ is the observed, blurred, noisy image, and $b_{c}(s)$ is the noiseless blurred image.

In particular, if it is assumed that $a(s, t)$ is spatially-invariant, that is, its effect depends only on the spatial distance between $s$ and $t$, then the preceding equation represents a convolution integral, where $a(s, t)=a(s-t)$. In this case, $b_{c}(s)$ is the result of convolving $a(s)$ and $x(s)$.

Since recording devices make only a finite number of measurements, the imaging model can be discretized and Equation (1.1) can be written as a matrix equation. The discretized model is:

$$
A x=b+\mu .
$$

${ }^{*}$ Manuscript date: September 25, 2001. This work was supported in part by the National Science Foundation under grant CCR-97-32022 and by the Office of Naval Research under grant N000140110181.

${ }^{\dagger}$ Applied Mathematics and Scientific Computation Program, University of Maryland, College Park, MD 20742 (armin@math.umd.edu)

‡Department of Computer Science and Institute for Advanced Computer Studies, University of Maryland, College Park, MD 20742. (oleary@cs.umd.edu). 
where the matrix $A$ is the discretized counterpart of $a(s, t)$, and $x$ and $b$ are also the discretized versions of the corresponding continuous functions. If the blurring function $a$ is assumed to be spatially-invariant, then the matrix $A$ has a special structure: for 1-dimensional signals it is Toeplitz and for 2-dimensional signals block Toeplitz with Toeplitz blocks.

If the cause of the blur and hence $a$ is not known exactly, then our estimate of $A$ has errors and the problem is known as blind deconvolution. In this case the model in (1.2) should be replaced by

$$
(A+E) x=b+r
$$

a problem of the total least norm variety. If the matrix $A$ has no special structure and the error $\|[E \mid r]\|_{p}$ is measured using the Frobenius norm, then the problem can be solved using the total least squares (TLS) method [5]. For image processing problems, the matrix $A$ has a special structure, and it is desirable to enforce the same structure on the error matrix E. Rosen, Park and Glick [22] developed the structured total least norm (STLN) method to solve such problems.

While STLN is useful for many structured linear problems, the blind deconvolution problem as encountered for image deblurring is generally ill-posed [9]. In particular, the matrix $A$ is often ill-conditioned, resulting in poor recovered images when STLN is applied.

Regularization methods must be implemented in order to stabilize STLN and to obtain useful results. In this paper it is shown how to implement Tikhonov regularization $[20,24]$ to arrive at the regularized structured total least norm (RSTLN) algorithm. While implementations of Tikhonov regularization for constrained total least squares problems had been developed previously [15, 17], the first even before the work of Rosen et. al. on the simpler problem, they focused solely on the 2 -norm case. The contributions herein are the extension for $p=1$ and $p=\infty$ norms and the comparison of methods. In the $p=1$ and $p=\infty$ cases, the main computational task lies in solving a linear program (LP).

The paper is structured as follows: in the next section the STLN method is introduced and derived. In $\S 3$ the general RSTLN method is introduced and derivations are presented for the $p=1,2$ and $\infty$ cases. Finally, in $\S 4$ we present numerical results and in $\S 5$ draw conclusions.

2. Derivation of the STLN Method. In order to understand the RSTLN method, a brief derivation of STLN based on [22] is given. For a more thorough derivation, the reader is referred to [22] and [12].

2.1. Total Least Squares and Structured Total Least Norm. The total least squares [5] formulation for solving problems as in Equation (1.3) is to find a matrix $E$ and a vector $r$ such that

$$
\|[E, r]\|_{F}
$$

is minimized, where $F$ denotes the Frobenius norm and $r=b-(A+E) x$ is the residual. If the matrix $A$ has a special structure which the user wants to enforce on $E$, then the TLS formulation is not applicable. Instead, the STLN formulation proves useful.

As in [22] assume that the matrix $A \in \mathbf{R}^{m \times n}$ is parameterized by elements of the vector $\alpha \in \mathbf{R}^{q}, q<m n$. Then the residual is a function of $\alpha$ and $x$. Hence, the 
STLN formulation is to find vectors $\alpha$ and $x$ such that

$$
\left\|\begin{array}{c}
r(\alpha, x) \\
D \alpha
\end{array}\right\|_{p}
$$

is minimized where $p=1,2$ or $\infty$ and $D$ is a diagonal weighting matrix through which the size of $\alpha$ is measured.

We assume that there is a relationship between $E$ and $x$. In particular, assume there exists a matrix $X$ parameterized by $x$ such that

$$
X \alpha=E x \text {. }
$$

For a detailed description on construction of the matrix $X$, the user is referred to [22] and [12], although the reader should note that if the matrix $E$ is structured then so is $X$.

Now let $\Delta x$ and $\Delta E$ denote small changes in $x$ and $E$, respectively, then

$$
X \Delta \alpha=(\Delta E) x \text {. }
$$

If we expand $r(\alpha, x)$ in a Taylor series about $\left[\alpha^{T} x^{T}\right]^{T}$ and ignore second order and higher terms, we have

$$
\begin{aligned}
r(\alpha+\Delta \alpha, x+\Delta x) & \approx b-(A+E) x-X \Delta \alpha-(A+E) \Delta x \\
& =r(\alpha, x)-X \Delta \alpha-(A+E) \Delta x .
\end{aligned}
$$

Hence, we have a linearization of (2.2):

$$
\min _{\Delta \alpha, \Delta x}\left\|\left[\begin{array}{cc}
X & A+E \\
D & 0
\end{array}\right]\left(\begin{array}{c}
\Delta \alpha \\
\Delta x
\end{array}\right)+\left(\begin{array}{c}
-r \\
D \alpha
\end{array}\right)\right\|_{p} .
$$

The general idea behind the STLN method is to start with some initial estimates for $x$ and $E$, solve the minimization problem in Equation (2.6) for $\Delta \alpha$ and $\Delta x$, set $x=x+\Delta x$ and $\alpha=\alpha+\Delta \alpha$, and update the residual $r$ and the matrices $E$ and $X$. The procedure is repeated iteratively until $\|\Delta \alpha\|$ and $\|\Delta x\|$ are below a specified tolerance, at which point the algorithm has converged to a solution. For a detailed description the reader is referred to [22].

3. Derivation of RSTLN. In order to make STLN more robust in the presence of noise (as is encountered in most image deblurring applications), a form of regularization must be introduced. The method of Tikhonov [24] is implemented herein, which prevents the solution $x$ from getting too large. In particular, Equation (2.2) can be modified to arrive at the Regularized Structured Total Least Norm (RSTLN) algorithm. The new problem formulation is to find vectors $\alpha$ and $x$ so that

$$
\left\|\begin{array}{c}
r(\alpha, x) \\
D \alpha \\
\lambda x
\end{array}\right\|_{p}
$$

is minimized, where $\lambda$ is a positive scalar known as the regularization parameter and $p=1,2$ or $\infty$. Using the relation in Equation (2.5) and similar reasoning as for the STLN method, the linearization of Equation (3.1) results in:

$$
\min _{\Delta \alpha, \Delta x}\left\|\left[\begin{array}{cc}
X & A+E \\
D & 0 \\
0 & \lambda I
\end{array}\right]\left(\begin{array}{c}
\Delta \alpha \\
\Delta x
\end{array}\right)+\left(\begin{array}{c}
-r \\
D \alpha \\
\lambda x
\end{array}\right)\right\|_{p} .
$$




\section{RSTLN Algorithm}

1. Set $E=0_{m \times n}$ and $\alpha=0_{q \times 1}$.

2. Compute $x$ by $\min _{x}\|A x-b\|_{p}$ (for $p=2$ this is just least squares).

3 . Compute $X$ from $x$ and the residual $r=b-A x$.

4. For $k=1,2, \ldots$ until $\|\Delta x\|,\|\Delta \alpha\| \leq \epsilon$ repeat Steps $4.1-4.3$

4.1. Solve

$$
\min _{\Delta \alpha, \Delta x}\left\|\left[\begin{array}{cc}
X & A+E \\
D & 0 \\
0 & \lambda I
\end{array}\right]\left(\begin{array}{c}
\Delta \alpha \\
\Delta x
\end{array}\right)+\left(\begin{array}{c}
-r(\alpha, x) \\
D \alpha \\
\lambda x
\end{array}\right)\right\|_{p} .
$$

4.2. Set $x=x+\Delta x$ and $\alpha=\alpha+\Delta \alpha$.

4.3. Construct $E$ from $\alpha$, and $X$ from $x$ and compute $r=b-(A+E) x$.

5. The recovered image is $x$ and the recovered blurring matrix $(\mathrm{A}+\mathrm{E})$.

TABLE 3.1

The general RSTLN algorithm (for arbitrary norm $p$ ) is listed in Table 3.1.

3.1. RSTLN for $p=2$. The minimization problem in the RSTLN formulation is equivalent to minimizing the function:

$$
\phi(\alpha, x)=\frac{1}{2}\|r(\alpha, x)\|_{2}^{2}+\frac{1}{2}\|D \alpha\|_{2}^{2}+\frac{1}{2}\|\lambda x\|_{2}^{2} .
$$

The 2-norm case has the property of differentiability so that Gauss-Newton theory is applicable. Using similar reasoning as in [22] for the STLN method, it follows that Step 4.1 is a Gauss-Newton method which approximates the Hessian of $\phi(\alpha, x)$ by the positive definite matrix $M^{T} M$, where

$$
M=\left[\begin{array}{cc}
X & A+E \\
D & 0 \\
0 & \lambda I
\end{array}\right]
$$

See also [3].

The least squares normal equations can be solved using the conjugate gradient method, where the Toeplitz (or block Toeplitz with Toeplitz block) structure of $M$ is exploited. In particular, the FFT is used for efficient computation of matrix-vector products.

Another more efficient approach for $p=2$ may be to apply the techniques of [14] for the non-regularized STLN to RSTLN. In particular, an algorithm based on the generalized Schur algorithm [16] for solving least squares problems is used which exploits the structure of the STLS matrix:

$$
\left[\begin{array}{cc}
X & A+E \\
D & 0
\end{array}\right]
$$


Since the RSTLN matrix $M$ has a similar structure to this, the method in [14] should be applicable. This may be the focus of future work.

3.2. RSTLN for $p=\infty$. For both the $p=1$ and $p=\infty$ cases Step 4.1 of the RSTLN algorithm is a linear program. To see this, an approach similar to [22] is used.

Let us first consider the derivation for $p=\infty$. Suppose the original image in vector form is $x \in \mathbf{R}^{\mathbf{n} \times \mathbf{1}}$, that $\alpha \in \mathbf{R}^{\mathbf{q} \times \mathbf{1}}$ and that the residual $r \in \mathbf{R}^{\mathbf{m} \times \mathbf{1}}$. Then the optimal function value in Step 4.1 is $\bar{\sigma}$, where $\bar{\sigma}$ is determined from the linear program

$$
\begin{aligned}
& \min _{\Delta \alpha, \Delta x, \bar{\sigma}} \bar{\sigma} \\
& \text { subject to }-\bar{\sigma} e_{m} \leq X \Delta \alpha+(A+E) \Delta x-r \leq \bar{\sigma} e_{m} \\
& -\bar{\sigma} e_{q} \leq \quad D \Delta \alpha+D \alpha \leq \bar{\sigma} e_{q} \\
& -\bar{\sigma} e_{n} \leq \lambda \Delta x+\lambda x \leq \bar{\sigma} e_{n}
\end{aligned}
$$

where $e_{k} \in \mathbf{R}^{\mathbf{k} \times \mathbf{1}}$ is a vector of ones.

Using the matrix $M$ we can write the LP in standard form:

$$
\begin{aligned}
& \min _{\Delta \alpha, \Delta x, \bar{\sigma}} \bar{\sigma} \\
& \text { subject to }\left[\begin{array}{cc}
M & -e_{m+n+q} \\
-M & -e_{m+n+q}
\end{array}\right]\left(\begin{array}{c}
\Delta \alpha \\
\Delta x \\
\bar{\sigma}
\end{array}\right) \leq\left(\begin{array}{c}
r \\
-D \alpha \\
-\lambda x \\
-r \\
D \alpha \\
\lambda x
\end{array}\right)
\end{aligned}
$$

Depending on the method to solve the LP, it may be useful to consider the dual formulation. Setting $\sigma=-\bar{\sigma}$ it follows that the dual is:

$$
\begin{aligned}
& \min _{y_{i} \geq 0} \quad r^{T} y_{1}-\alpha^{T} D y_{2}-\lambda x^{T} y_{3}-r^{T} y_{4}+\alpha^{T} D y_{5}+\lambda x^{T} y_{6} \\
& \text { subject to } \quad\left[\begin{array}{cc}
M^{T} & -M^{T} \\
e_{m+n+q}^{T} & e_{m+n+q}^{T}
\end{array}\right]\left(\begin{array}{c}
y_{1} \\
y_{2} \\
y_{3} \\
y_{4} \\
y_{5} \\
y_{6}
\end{array}\right) \leq\left(\begin{array}{c}
0 \\
0 \\
0 \\
\vdots \\
0 \\
1
\end{array}\right)
\end{aligned}
$$

where $y_{1}, y_{3} \in \mathbf{R}^{m \times 1}, y_{2}, y_{4} \in \mathbf{R}^{q \times 1}$, and $y_{3}, y_{6} \in \mathbf{R}^{n \times 1}$. The system in Equation (3.8) can be solved using any standard simplex or interior point method.

The reader should note that since the matrix $M$ has a special structure (Toeplitz or block Toeplitz with Toeplitz blocks), any practical implementation of RSTLN for $p=1$ or $p=\infty$ should exploit this property when solving the LP.

3.3. RSTLN for $p=1$. The derivation for the $p=1$ case is similar to the $p=\infty$ case. Again, let $\bar{\sigma}$ be the optimal function value in Step 4.1. In particular, assuming $x, \alpha$, and $r$ are defined as previously, we have that $\bar{\sigma}$ is determined by 


$$
\begin{array}{cccc}
\min _{\Delta \alpha, \Delta x, \bar{\sigma}} \bar{\sigma}= & \sum_{i=1}^{m} \bar{\sigma}_{1_{i}}+\sum_{i=1}^{q} \bar{\sigma}_{2_{i}}+\sum_{i=1}^{n} \bar{\sigma}_{3_{i}} & \\
\text { subject to } & -\bar{\sigma}_{1} \leq X \Delta \alpha+(A+E) \Delta x-r & \leq \bar{\sigma}_{1} \\
& -\bar{\sigma}_{2} \leq & D \Delta \alpha+D \alpha & \leq \bar{\sigma}_{2} \\
& -\bar{\sigma}_{3} \leq & \lambda \Delta x+\lambda x & \leq \bar{\sigma}_{3}
\end{array}
$$

where $\bar{\sigma}_{1} \in \mathbf{R}^{m \times 1}, \bar{\sigma}_{2} \in \mathbf{R}^{q \times 1}$, and $\bar{\sigma}_{3} \in \mathbf{R}^{n \times 1}$. Using the matrix $M$ we can write the LP as:

$$
\begin{aligned}
& \min _{\Delta \alpha, \Delta x, \bar{\sigma}} \bar{\sigma}=\sum_{i=1}^{m} \bar{\sigma}_{1_{i}}+\sum_{i=1}^{q} \bar{\sigma}_{2_{i}}+\sum_{i=1}^{n} \bar{\sigma}_{3_{i}} \\
& \text { subject to }\left[\begin{array}{cc}
M & -I_{m+n+q} \\
-M & -I_{m+n+q}
\end{array}\right]\left(\begin{array}{c}
\Delta \alpha \\
\Delta x \\
\bar{\sigma}_{1} \\
\bar{\sigma}_{2} \\
\bar{\sigma}_{3}
\end{array}\right) \leq\left(\begin{array}{c}
r \\
-D \alpha \\
-\lambda x \\
-r \\
D \alpha \\
\lambda x
\end{array}\right)
\end{aligned}
$$

As for the $p=\infty$ case, the user may want to use the dual formulation. Setting $\sigma=-\bar{\sigma}$, then our formulation becomes

$$
\begin{aligned}
& \min _{y_{i} \geq 0} r^{T} y_{1}-\alpha^{T} D y_{2}-\lambda x^{T} y_{3}-r^{T} y_{4}+\alpha^{T} D y_{5}+\lambda x^{T} y_{6} \\
& \text { subject to }\left[\begin{array}{cc}
M^{T} & -M^{T} \\
I_{m+n+q} & I_{m+n+q}
\end{array}\right]\left(\begin{array}{l}
y_{1} \\
y_{2} \\
y_{3} \\
y_{4} \\
y_{5} \\
y_{6}
\end{array}\right) \leq\left(\begin{array}{c}
0_{m \times 1} \\
0_{q \times 1} \\
0_{n \times 1} \\
e_{m} \\
e_{q} \\
e_{n}
\end{array}\right)
\end{aligned}
$$

where all $y_{i}$ are as defined previously for the $\infty$-norm case, and $0_{k \times 1}$ is a vector of zeros.

3.4. Convergence of RSTLN for $p=1$ or $p=\infty$. As for the STLN problem, the function minimized in Equation (3.1) is nonconvex so that there is no guarantee that the RSTLN algorithm converges to a global minimum. For the $p=2$ norm case Gauss-Newton theory is applicable: a suitable line search method (see for example [3]) can be used to guarantee convergence to a local minimizer from any starting point.

For $p=1$ and $p=\infty$ Gauss-Newton theory is not directly applicable since differentiability is lost. But the essential idea is the same as for the $p=2$ norm. In particular, the solutions $\left[\begin{array}{ll}\Delta \alpha^{T} & \Delta x^{T}\end{array}\right]^{T}$ to the LPs given in Equations (3.7) and (3.10) can be thought of as descent directions to the function in Equation (3.1) for the respective $p$-norm. Again, in order to guarantee convergence to a local minimizer from any starting point, a line search method can be implemented.

4. Numerical Results. In this section, experiments will be given which show that RSTLN deblurs images better than the STLN method. In particular, examples are shown comparing RSTLN and STLN for the $p=1,2$ and $\infty$ norms. We also compare our results with other blind deconvolution algorithms. 


\subsection{Experimental Design.}

4.1.1. Numerical Issues. All of our code was written in MATLAB to take advantage of its image visualization capabilities.

The choice of regularization parameter is a well-studied problem (see, for example, [8]). Ideally, the choice balances the need to stay close to the original noisecontaminated problem [10] without causing its ill-conditioning to produce unacceptable noise in the solution. In our experiments, we were concerned with the best solution obtainable for any choice of parameter. We set $D=I$ and solved each problem for a wide range of values $\lambda>0$, choosing the parameter resulting in the smallest value for the 2-norm of the image error.

For the STLN and RSTLN algorithms, a linear problem needs to be solved at each iteration; see Step 4.1 of Table 3.1. For the $p=2$ norm, we used the conjugate gradient least squares method to solve this problem. We set the CG termination condition to a relative residual tolerance of $10^{-6}$ or 1000 iterations. This generally produces satisfactory accuracy to determine the descent direction, but for larger images, the maximum number of iterations was sometimes taken.

We stop the STLN or RSTLN iterations when

$$
\begin{gathered}
\|\Delta x\|_{2} /\|b\|_{2}<t o l \\
\left\|A_{\Delta \alpha}\right\|_{2} /\left\|A_{e s t}\right\|_{2}<t o l
\end{gathered}
$$

where $b$ is the blurred image, $A_{\text {est }}$ the blurring matrix parameterized by the initial PSF estimate, and $A_{\Delta \alpha}$ the blurring matrix parameterized by the current PSF error $\Delta \alpha$.

For the $p=1$ and $p=\infty$ cases we solved the linear program in Step 4.1 using the MATLAB function linprog. $m$ with the largescale model employed. The function uses the LIPSOL [25] algorithm and is based on a primal-dual interior point method. Because of limitations in the MATLAB interface to LIPSOL, we were only able to set our stopping criteria to $O\left(10^{-2}\right)$ to $O\left(10^{-3}\right)$ compared to tolerances of $O\left(10^{-6}\right)$ for the STLN experiments in [22]; a smaller tolerance caused LIPSOL to fail to converge. Even with this difficulty, RSTLN gives better results than STLN.

Our current implementation is restricted to fairly small images because of the large number of constraints in the linear program. While the actual constraint matrix $M$ passed into linprog.m is sparse, its factorization within the routine generally is not. Hence, the LP solver as implemented in MATLAB is very memory intensive and currently restricts our test cases to images no larger than $100 \times 100$.

We computed the following values in order to compare STLN and RSTLN:

$$
\begin{aligned}
b_{\text {pert }} & =\left\|b_{\text {noisy }}-b_{\text {true }}\right\|_{2} /\left\|b_{\text {true }}\right\|_{2} \\
A_{\text {pert }} & =\left\|A_{\text {noisy }}-A_{\text {true }}\right\|_{F} /\left\|A_{\text {true }}\right\|_{F} \\
x_{\text {err }} & =\left\|x_{\text {rec }}-x_{\text {true }}\right\|_{2} /\left\|x_{\text {true }}\right\|_{2} \\
A_{\text {err }} & =\left\|(A+E)_{\text {rec }}-A_{\text {true }}\right\|_{F} /\left\|A_{\text {true }}\right\|_{F} \\
b_{\text {err }} & =\left\|b_{\text {rec }}-b_{\text {true }}\right\|_{2} /\left\|b_{\text {true }}\right\|_{2}
\end{aligned}
$$

where $x_{r e c}$ is the recovered image (in vector form), $(A+E)_{r e c}$ represents the blurring matrix parameterized by the recovered PSF, $b_{r e c}$ is the result obtained from convolving the recovered image and PSF, and $x_{\text {true }}, A_{\text {true }}$, and $b_{\text {true }}$ are the correct values, so that $A_{\text {true }} x_{\text {true }}=b_{\text {true }}$. The values $b_{\text {pert }}$ and $A_{\text {pert }}$ represent the perturbed versions of $b$ and $A$, where $b_{\text {noisy }}$ and $A_{\text {noisy }}$ are the noisy versions of $b$ and $A$ with the addition of zero-mean Gaussian noise. 
4.1.2. Comparison with Other Blind Deconvolution Methods. We compare RSTLN with two other blind deconvolution methods: blind Lucy-Richardson, and the APEX/SECB method of Carasso.

The blind Lucy-Richardson algorithm is an extension of the well-known original Lucy-Richardson method [13, 21]. The original iterative method was derived from Bayes' Theorem and assumes that the blurred image, the original image, and the PSF are (possibly non-normalized) probability density functions. The most common and efficient implementation makes use of the FFT to compute convolutions. This implicitly imposes periodic boundary conditions on the image.

The blind version is similar to the original method; each iteration alternately uses several iterations of the non-blind algorithm to estimate a new PSF and then a new image. It is generally more effective for images having many pixels and for images with fewer sharp edges, since convolution tends to smooth edge boundaries [9].

The algorithm can be used without FFTs, but it is computationally much slower and may produce ringing if the image does not have finite support. But because the method has a probabilistic basis, any implementation must conserve energy. Thus, a non-periodic (for example, zero boundary condition) implementation is useful only for images having support strictly inside the image boundaries. Convolutions involving images with non-finite support do not conserve energy and result in data being lost outside of the original image boundary; this leads to ringing in the resulting images.

The stopping criterion for MATLAB's blind Lucy-Richardson function deconvblind.m is based solely on the input number of iterations. The user may specify this total number of iterations or use the default value of 10 . Our non-FFT implementation is similar to the non-blind MATLAB routine deconvlucy.m, but lets the user specify the total number of iterations and, for each, the number of Lucy-Richardson inner iterations to update the image and PSF estimates. We estimate the optimal number of iterations by recovering images using a wide variety of choices and then choosing the image resulting in the smallest 2-norm error. For our comparison test cases, where our goal was to show only general trends in the recovered images, we often used a default of 10 iterations, modifying this number as needed.

Carasso's APEX/SECB method [1] can be applied to the class of PSFs $a$ whose FFT, denoted by $\hat{a}(\xi, \eta)$, is of the form:

$$
\hat{a}(\xi, \eta)=e^{-\alpha\left(\xi^{2}+\eta^{2}\right)^{\beta}}
$$

where $\xi$ and $\eta$ are the respective frequency coordinates. If the blurred image $b=a \otimes x$ is obtained by (periodic) convolution, then in the Fourier domain

$$
\begin{aligned}
\hat{b}(\xi, \eta) & =\hat{x}(\xi, \eta) \cdot \hat{a}(\xi, \eta) \\
& =\hat{x}(\xi, \eta) \cdot e^{-\alpha\left(\xi^{2}+\eta^{2}\right)^{\beta}} .
\end{aligned}
$$

The idea behind the PSF identification method is to fit the function $\alpha|\xi|^{2 \beta}$ to the logarithm of the Fourier transform of the blurred image and an estimate of the true image; see [1] for details. If the image or the PSF fails to meet necessary requirements, then such a fit will not be possible.

4.2. Test 1. Our first test consists of a cross of size $21 \times 21$. The true PSF is a Gaussian blur with variance 2.5 , truncated to a support of size $11 \times 11$.

The blurred image was obtained by convolving the original image and PSF, assuming that pixel values outside the image are zero (zero boundary conditions). The 


\begin{tabular}{|l|c|c|c|}
\hline Test Case 1 & $x_{\text {err }}$ & $A_{\text {err }}$ & $b_{\text {err }}$ \\
\hline$p=2$ STLN & 1.19 & $3.97 \mathrm{e}-2$ & $1.1 \mathrm{e}-3$ \\
$p=2$ RSTLN & 0.39 & $4.10 \mathrm{e}-2$ & $1.1 \mathrm{e}-3$ \\
\hline$p=1$ STLN & 0.97 & $3.99 \mathrm{e}-2$ & $1.4 \mathrm{e}-3$ \\
$p=1$ RSTLN & 0.44 & $4.00 \mathrm{e}-2$ & $1.1 \mathrm{e}-2$ \\
\hline$p=\infty$ STLN & 0.50 & $4.02 \mathrm{e}-2$ & $5.5 \mathrm{e}-1$ \\
$p=\infty$ RSTLN & 0.45 & $3.98 \mathrm{e}-2$ & $4.9 \mathrm{e}-1$ \\
\hline \multicolumn{4}{|c}{ TABLE 4.1}
\end{tabular}

RSTLN Errors for $p=1,2$ and $\infty$. We list the errors in the image $x$, the matrix $A$ and the residual error $b_{\text {err }}$ for the unregularized STLN and the RSTLN methods for each of the norms. For the $p=1$ and $p=2$ norms the $R S T L N$ recovered image error $x_{e r r}$ is much smaller than for STLN. For $p=\infty$ the image error is near optimal and the error using RSTLN is only slightly smaller than for $S T L N$.

original and blurred images are shown in (A) and (B) of Figure 4.1. Random, zero mean 6-bit noise was added to the PSF to obtain the initial PSF estimate. This resulted in $A_{\text {pert }}=3.99 \times 10^{-2}$. Furthermore, 11-bit Gaussian noise was added to the blurred image, resulting in $b_{\text {pert }}=1.10 \times 10^{-3}$.

The errors resulting from the STLN and RSTLN methods for the different pnorms are shown in Table 4.1. The corresponding images are shown in Figure 4.1, (C) through $(\mathrm{H})$. From the error table we see that the use of RSTLN generally increases the error $A_{e r r}$ in the blurring matrix and the residual error $b_{e r r}$. For the 1- and 2- norms, however, the error $x_{e r r}$ in the image estimate is considerably lower, so the reconstructed image is improved. For the $p=\infty$ norm, the image obtained from STLN was near optimal, and all RSTLN experiments for nonzero values of the regularization parameter $\lambda$ resulted in higher image errors.

In Figure 4.2 we present the results of the blind Lucy-Richardson method (denoted by LR). In (A) we show results obtained by LR in reconstructing images blurred with periodic boundary conditions (6-bit noise added), using 20 outer iterations with 10 Lucy-Richardson iterations in each. The width of the cross is broadened due to blurring of the edges during the reconstruction.

In Figure 4.2 (B) through (F), we present the result of various attempts to reconstruct the image with zero boundary conditions from Figure 4.1. In (B) we show the result obtained by using 5 outer iterations with 10 Lucy-Richardson iterations each, computing convolutions using zero padded images. It is clear that the image is distorted and ringing is observed throughout. The other images are reconstructed using the MATLAB-supplied implementation of blind Lucy-Richardson, which we call MLR. In (C) we show the M-LR result, beginning with the blur estimate as for RSTLN, and stopping after the MATLAB-default 10 iterations. We repeat this experiment in (D), but starting from a flat PSF estimate (a matrix of ones of size $11 \times 11$ ). In both cases only poor reconstructions are obtained. In (E) and (F) we show similar results as in (C) and (D), except that the image was tapered using edgetaper.m, which seeks to transform a nonperiodic image into a more periodic one by reblurring the edges of an image with a suitable PSF. The reader is referred to [23] for details. We performed 50 and $100 \mathrm{M}-\mathrm{LR}$ iterations, respectively. The reader should note that the algorithm is not able to reconstruct data near the image boundary, although the interior is adequately recovered.

The APEX/SECB method cannot be applied to this image, because it is too small to yield enough data points. 


\begin{tabular}{|l|c|c|c|}
\hline Test Case 2 & $x_{\text {err }}$ & $A_{\text {err }}$ & $b_{\text {err }}$ \\
\hline$p=2$ STLN & 4.2895 & $4.03 \mathrm{e}-2$ & $1.03 \mathrm{e}-2$ \\
$p=2$ RSTLN & 0.5885 & $1.15 \mathrm{e}+0$ & $9.20 \mathrm{e}-3$ \\
\hline \multicolumn{4}{r}{ TABLE 4.2}
\end{tabular}

$R S T L N$ Errors for $p=2$ for the large cross test case. We list the errors in the image $x$, the matrix $A$ and the residual error $b_{\text {err }}$ for the unregularized STLN and the RSTLN methods for $p=2$. For the $\operatorname{RSTLN}(\lambda=2.5)$ recovered image error $x_{e r r}$ is much smaller than for STLN.

Test 2. Our next test consists of a somewhat broader cross image of size $41 \times 41$ with a nonzero cross width of 5 . The image was blurred with an $11 \times 11$ Gaussian. Gaussian 8-bit noise was added to the blurred images, resulting in $b_{\text {pert }}=1.05 \times 10^{-2}$ and $9.8 \times 10^{-3}$, respectively. The blur estimate was obtained by adding 6 -bit noise to the original blur, resulting in $A_{\text {pert }}=3.91 \times 10^{-2}$.

Again, we present results comparing the STLN, RSTLN, LR, and M-LR methods, as well as Carasso's APEX/SECB method. In Figure 4.3 we show the original and blurred images in (A) and (B). In (C) we show the STLN 2-norm solution (that is, without any regularization) and in (D) the best RSTLN 2-norm solution with regularization (using $\lambda=0.75$ ). (The RSTLN $p=1$ and $p=\infty$ were not computed due to the expense of solving the linear programming problems.) The resulting STLN and RSTLN errors for the 2-norm are shown in Table 4.2.

For APEX/SECB, the original image in (A) was blurred using periodic boundary conditions as in Equation (4.2) using parameters $\alpha=0.075$ and $\beta=1$. This resulted in a blurred image nearly identical to (B). Again, 8-bit noise was added to the blurred image. In subplot (E) we show the results of using APEX/SECB for PSF identification and subsequent deblurring of the periodic noisy blurred image. The APEX PSF identification procedure resulted in parameter estimates of $\alpha_{\text {est }}=0.0749$ and $\beta_{\text {est }}=$ 0.9756 , which are fairly close to the true parameter values. Unfortunately, this method was unsuccessful for images blurred with zero boundary conditions and noise added. In (F), we show the APEX optimization function for different scalar value image estimates. The non-smooth family of curves corresponds to the optimization function for different scalar estimates for the unknown image quantity $\log |\hat{f}(\xi, 0)|$ if the natural logarithm is applied to the right and left hand sides in Equation 4.2 and when a noisy zero boundary condition blurred image is used. The curves do not have the proper form and thus do not permit a curve fit of the form $\alpha|\xi|^{2 \beta}$. For this case no proper PSF can be found.

In Figure 4.4 we present results of the blind Lucy-Richardson algorithm. In (A) we see that the algorithm gives a good result for periodic blurs, but the reconstruction for a zero boundary condition exhibits ringing and distortion. These results used 50 outer iterations, each using 10 Lucy-Richardson iterations. In (B) we give the result for the zero boundary condition image using the zero boundary implementation. We then apply the M-LR algorithm to a noisy zero boundary blurred image. In (C) and (D) we show results using no tapering, 25 iterations, and using an initial guess of either the RSTLN blur estimate or a matrix of ones of size $11 \times 11$. Both results exhibit ringing due to improper boundary conditions. In (E) and (F) we show M-LR results with tapering, using 10 outer iterations and initial blur estimates as in (C) and (D). The reconstructions are not useful.

4.3. Test 3. Our final comparison test consists of an image obtained from the NASA Image Exchange (http://nix.nasa.gov). It shows the corona of the sun and 


\begin{tabular}{|l|c|c|c|}
\hline Test Case 3 & $x_{e r r}$ & $A_{\text {err }}$ & $b_{\text {err }}$ \\
\hline$p=2$ STLN & 20.01 & $2.47 \mathrm{e}-2$ & $2.19 \mathrm{e}-2$ \\
$p=2$ RSTLN & 0.9265 & $3.8483 \mathrm{e}+0$ & $6.71 \mathrm{e}-1$ \\
\hline \multicolumn{4}{|c|}{ TABLE 4.3}
\end{tabular}

RSTLN Errors for $p=2$ for the sun test case. We list the errors in the image $x$, the matrix $A$ and the residual error $b_{e r r}$ for the unregularized $S T L N$ and the RSTLN methods for $p=2$. For the $\operatorname{RSTLN}(\lambda=75)$ recovered image error $x_{e r r}$ is much smaller than for STLN.

a large solar eruption. We truncated the image to size $99 \times 99$ and reduced it to gray-scale.

Again, the image was blurred with a Gaussian PSF of size $11 \times 11$ in two ways: one assuming zero values for pixels outside the image, and the other assuming a periodic image. A 6-bit noisy version of the zero boundary condition blurred image was obtained by adding zero mean Gaussian noise. This resulted in $b_{\text {pert }}=2.20 \times 10^{-2}$. For the periodic image no noise was added to the blurred image. The blur estimate was obtained by adding 6 -bit noise to the original blur $\left(A_{\text {pert }}=2.46 \times 10^{-2}\right)$.

In Figure $4.5(\mathrm{~A})$ we show the original and in (B) the noisy blurred image using zero boundary conditions. In (C) we show the STLN result using the 2-norm. Due to the high noise level in both the blurred image and the blur estimate, no useful result was obtained. In (D) we show the best result using the RSTLN method with a regularization value of $\lambda=75$. We remark that in this case the algorithm did not converge to a tolerance of $10^{-2}$. Instead we stopped prematurely after 10 iterations. A larger number of iterations which did achieve the desired tolerance produced an image of lesser quality contaminated by severe ringing.

In Table 4.3 we computed the resulting errors for the STLN and RSTLN methods. Although $A_{e r r}$ and $b_{e r r}$ are increased for RSTLN with respect to STLN, clearly the image error is drastically reduced using the RSTLN method.

For the APEX/SECB method the image was blurred with a Gaussian blur using periodic boundary conditions and parameters $\alpha=0.01$ and $\beta=1$ as in Equation (4.2). This resulted in a blurred image very similar to the one in (B). Noise (6-bit) was added to the blurred image. Using the APEX PSF identification method, a curve fit to the optimization function was done, resulting in parameter estimates of $\alpha_{e s t}=0.0108$ and $\beta_{\text {est }}=1.028$. These are fairly close to the true PSF parameters. In (E) we show the APEX/SECB recovered image using the noisy blurred image with periodic boundary conditions. In $(\mathrm{F})$ we show the function to be fit using the noisy image with zero boundary conditions. We plot the function using different scalar estimates for the original image component in Equation 4.2. None of the functions have the proper form and a suitable curve fit of the form $\alpha|\xi|^{2 \beta}$ is not possible. For this case no useful PSF was found.

In Figure 4.6 we show the results from the various Lucy-Richardson experiments. In subplot (A) we have the LR result using a periodic image using our own periodic LR implementation. We performed 10 iterations, each with 10 Lucy-Richardson iterations. In (B) we show the result using the zero boundary implementation and a zero boundary blurred image. We performed outer 15 iterations, each with 10 iterations to estimate the new PSF and image. Severe ringing is present. In (C) and (D) we show the non-tapered M-LR results using the RSTLN blur estimate, an $11 \times 11$ matrix of ones for the blur estimate and a zero boundary blurred image. 25 outer iterations were performed, with 10 iterations each. For the result in (C) ringing is observed near the image boundary, whereas in (D) the image is severely distorted. Finally, in 
(E) and (F) we obtained results using M-LR and a tapered noisy blurred image using the two different initial blur estimate types. For the result in (E) 25 iterations were performed which produced reasonable results. The result in $(\mathrm{F})$ was obtained after 10 iterations with less favorable results.

5. Conclusions. We have presented th RSTLN algorithm for blind deconvolution. Like the STLN method, RSTLN preserves any affine structure in the matrix, and the user has the choice of minimizing the error for the 2-norm or for other norms such as the 1 and $\infty$ norms. The use of norms other than the 2-norm leads to good image recovery, although the cost is substantially higher.

In contrast to other methods, such as that of Carasso's APEX/SECB, the RSTLN method does not depend on having a periodic image. Ringing in the reconstructed images is less of a problem. Therefore, we can apply the RSTLN method for arbitrary boundary conditions, for example zero (Dirichlet), Neumann (data outside of the image boundary is a reflection of the corresponding data inside), or periodic.

Acknowledgments. The authors wish to thank Cleve Moler and Bruce Golden for their help on linear programming and MATLAB, and Jon McCoy for spirited discussions on the Lucy-Richardson method.

\section{REFERENCES}

[1] A. S. Carasso, Direct blind deconvolution, SIAM J. Appl. Math., to be submitted, 1999.

[2] P. J. Davis, Circulant Matrices, Wiley, New York, 1979.

[3] R. Fletcher, Practical Methods of Optimization, Vol.2, Second ed., Wiley and Sons, New York, 1980.

[4] G. Golub, M. Heath, and G. Wahba, Generalized cross-validation as a method for choosing a good ridge parameter, Technometrics, 21, (1979), pp. 215-223.

[5] G. H. Golub and C. F. VAN LOAN, An analysis of the total least squares problem, SIAM J. Numer. Anal., 17, (1980), pp. 883-893.

[6] R. C. Gonzalez and P. Wintz, Digital Image Processing, Addison-Wesley, Reading, Massachusetts, 1977.

[7] P. C. Hansen, Analysis of discrete ill-posed problems by means of the L-curve, SIAM Review, 34, (1992), pp. 561-580.

[8] P. C. Hansen, Rank-Deficient and Discrete Ill-Posed Problems, SIAM Press, Philadelphia, 1998.

[9] P. C. Hansen, Numerical Aspects of Deconvolution, Lecture Notes, Department of Mathematical Modeling, Technical University of Denmark, 2000.

[10] M. E. KILMER AND D. P. O'LEARY, Choosing regularization parameters in iterative methods for ill-posed problems, SIAM J. Sci. Comp., to appear.

[11] R. L. Lagendijk and J. Biemond, Iterative Identification and Restoration of Images, Kluwer Academic Publishers, Norwell, Massachusetts, 1991.

[12] P. Lemmerling, N. Mastronardi, And S. Van Huffel, Fast algorithm for solving Hankel/Toeplitz structured total least squares problem, Numer. Alg. 21, (2000), pp. 371-392.

[13] L. B. LuCY, An iterative technique for the rectification of observed distributions, Astronomical Journal, 79, (1974), pp. 745-754.

[14] N. Mastronardi, P. Lemmerling, and S. Van Huffel, Fast structured total least squares algorithm for solving the basic deconvolution problem, SIAM J. Matrix Anal. Appl., 22, (2000), pp. 533-553.

[15] V. Mesarović, N. Galatsanos, and A. Katsaggelos, Regularized constrained total least squares image restoration, IEEE Transactions on Image Processing, 4, (1995), pp. 10961108.

[16] B. DE MOoR, Total least squares for affinely structured matrices and the noisy realization problem, IEEE Trans. Signal Process., 42 (1994), pp. 3004-3113.

[17] M. K. NG, R. J. Plemmons, and F. Pimentel, A new approach to constrained total least squares image restoration, Linear Algebra and its Appl., 316, (2000), pp. 237-258. 
[18] D. P. O'Leary, Near-Optimal Parameters for Tikhonov and Other Regularization Methods, Computer Science Department Report CS-TR-4004 Institute for Advanced Computer Studies Report UMIACS-TR-99-17, University of Maryland, March 1999.

[19] D. P. O'LEARY AND J. A. Simmons, A bidiagonalization-regularization procedure for large scale discretizations of ill-posed problems, SIAM J. Sci. Stat. Comp., 2, (1981), pp. 474-489.

$[20]$ D. L. PHILliPs, A technique for the numerical solution of certain integral equations of the first kind, J. Assoc. Comput. Mach., 9 (1962), pp. 84-97.

[21] W. H. Richardson, Bayesian-based iterative method of image restoration, J. Opt. Soc. Am. A, 62, (1972), pp. 55-59.

[22] J. B. Rosen, H. PARK AND J. GLick, Total least norm formulation and solution for structured problems, SIAM J. Matrix Anal. Appl., 17, (1996), pp. 110-126.

[23] J. Skilling And S. F. Gull, Algorithms and applications, in 1st Workshop on Maximum Entropy and Bayesian Methods in Inverse Problems, C. Ray Smith and W. T. Grandy, Jr., eds., D. Reidel Publishing Company, 1985, pp. 83-132.

[24] A. N. TikHonov, Solution of incorrectly formulated problems and the regularization method, Soviet Math. Dokl., 4 (1963), pp. 1035-1038; English translation of Dokl. Akad Nauk. SSSR, 51 (1963), pp. 501-504.

[25] Yin ZhANG, Solving large-scale linear programs by interior-point methods under the MATLAB environment, Technical Report, Department of Computational and Applied Mathematics, Rice University, Texas, 1997. 
(A) ORIGINAL

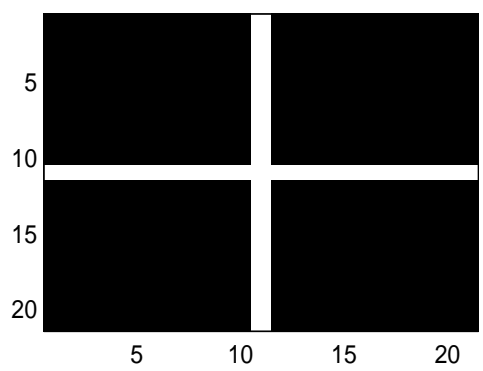

(C) STLN-inf

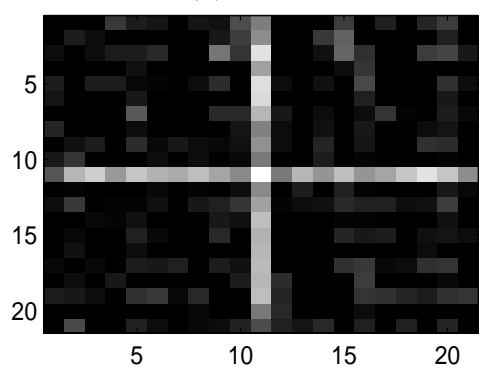

(E) STLN-2

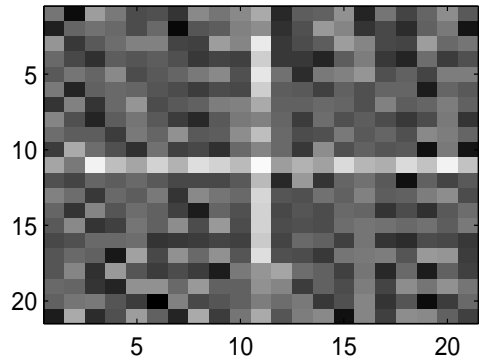

(G) STLN-1

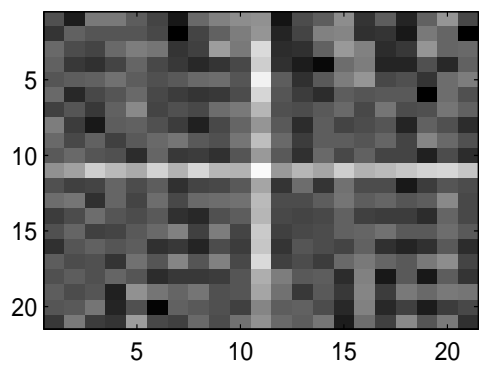

(B) NOISY BLURRED IMAGE (0 BC)

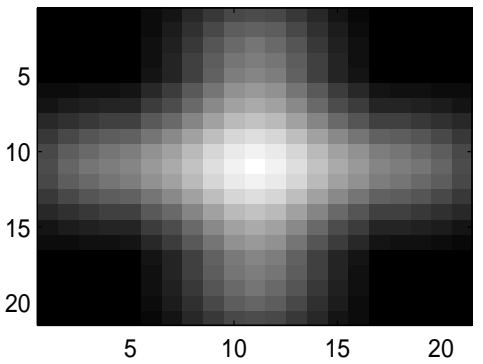

(D) RSTLN-inf

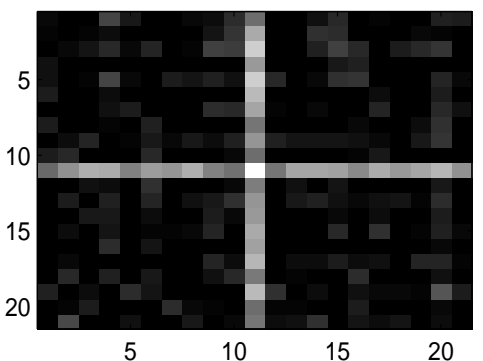

(F) RSTLN-2

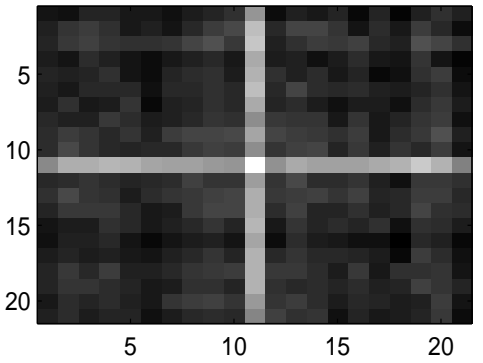

(H) RSTLN-1

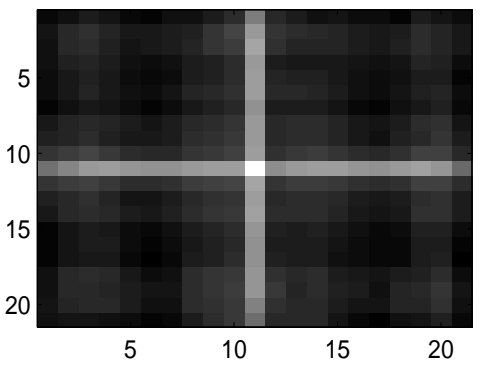

FIG. 4.1. RSTLN - Cross (noise, Gaussian blur). Test 1, results of STLN and RSTLN methods using $p=1,2, \infty$-norms. Random noise is present in the blurred image. The blur estimate is the true blur plus the addition of 6 -bit noise so that $A_{\text {pert }}=3.99 \times 10^{-2}$. Random, zero mean Gaussian 11-bit noise was added to the blurred image so that $b_{\text {pert }}=1.10 \times 10^{-3}$. (A) Original image - $21 \times 21$; (B) Noisy blurred image (zero BC); (C) STLN ( $\infty$-norm) solution with tol $=10^{-2}$. Solution is near optimal: 13 iterations; (D) RSTLN ( $\infty$-norm) recovered image with tol $=10^{-2}$, regularization parameter $\lambda=0.001$, 12 iterations. (E) STLN (2-norm) solution with tol $=10^{-3}$, 22 iterations; (F) RSTLN (2-norm) recovered image with tol $=10^{-3}, \lambda=0.05$, 27 iterations; $(G)$ $S T L N$ (1-norm) solution with tol $=10^{-2}, 13$ iterations; (H) RSTLN (1-norm) recovered image with tol $=10^{-2}, \lambda=0.5$, 50 iterations. 
(A) LR w/noise (PER. BC)

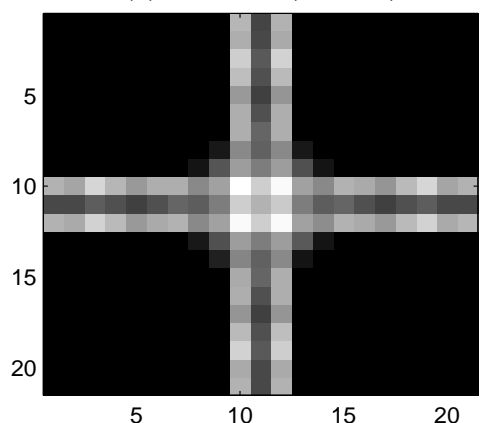

(C) M-LR w/noise (no taper, blur est.)

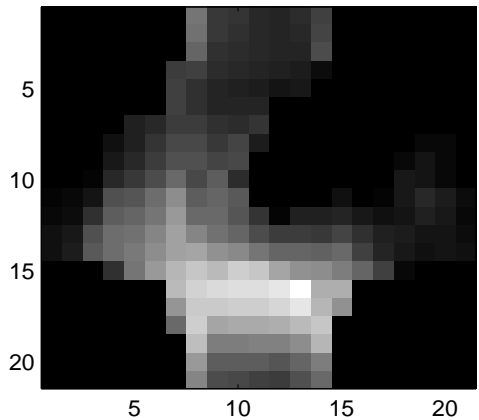

(E) M-LR w/noise (taper, blur est.)

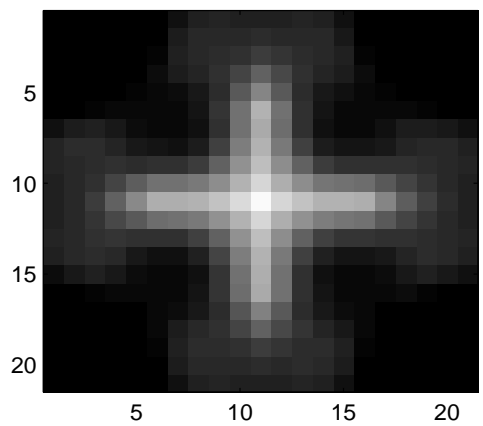

(B) LR w/noise (0 BC)

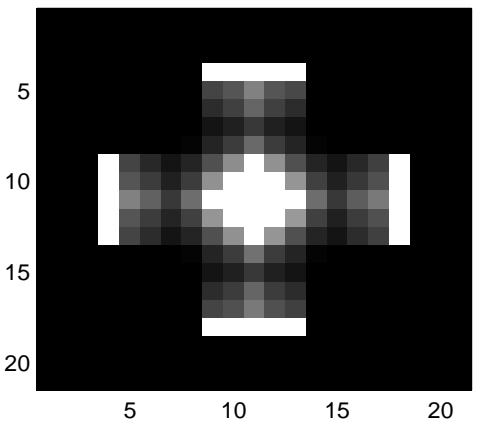

(D) M-LR w/noise (no taper, ones)

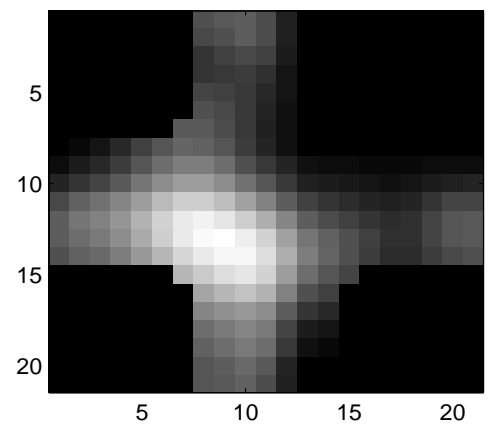

(F) M-LR w/noise (taper, ones)

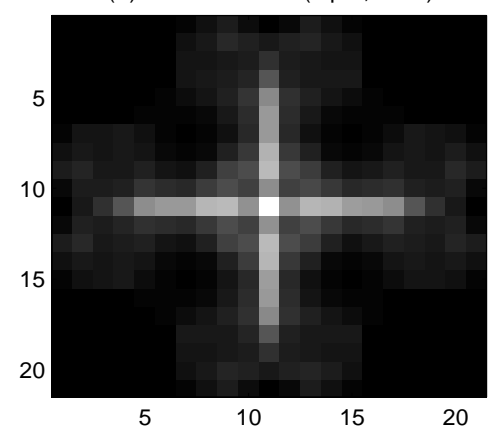

FIG. 4.2. Test 1, Lucy-Richardson results. (A) periodic LR implementation using a periodic blurred image, $20 \mathrm{LR}$ iterations each with 10 iterations; (B) zero boundary condition LR implementation using a zero BC blurred image, 5 LR iterations each with 10 iterations; $(C) M-L R$ result without tapering and using the RSTLN initial PSF estimate, 10 iterations; (D) M-LR result without tapering and using an $11 \times 11$ matrix of ones for the initial PSF estimate, 10 iterations; (E) M-LR result with tapering and using the RSTLN initial PSF estimate, 50 iterations; (F) $M-L R$ result with tapering and using an $11 \times 11$ matrix of ones for the initial PSF estimate, 100 iterations 
(A) ORIGINAL

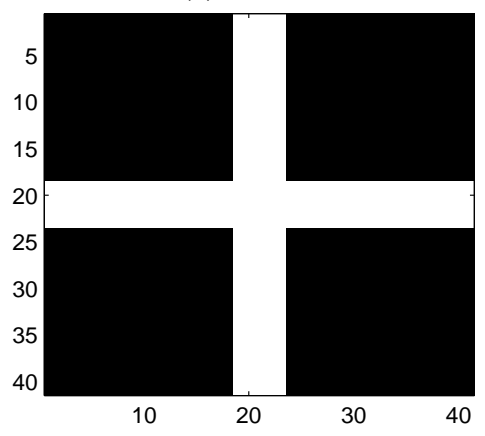

(C) STLN-2

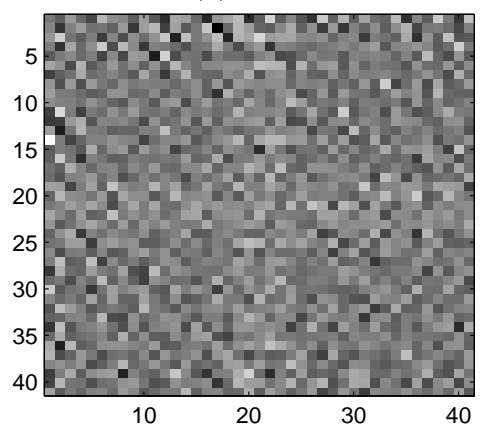

(E) APEX/SECB w/noise (PER. BC)

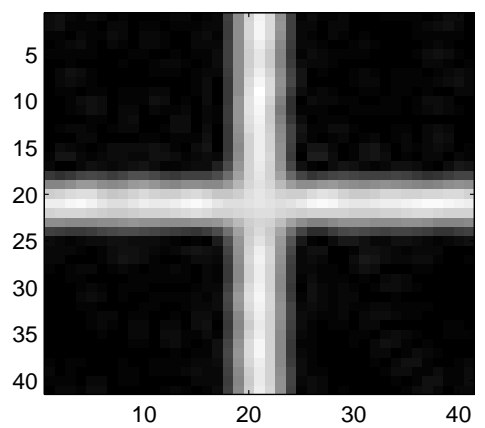

(B) NOISY BLURRED IMAGE (0 BC)

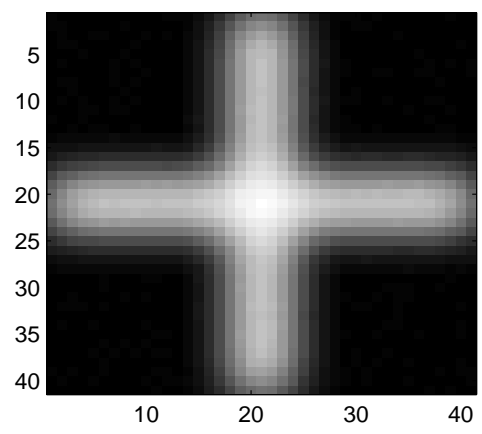

(D) RSTLN-2

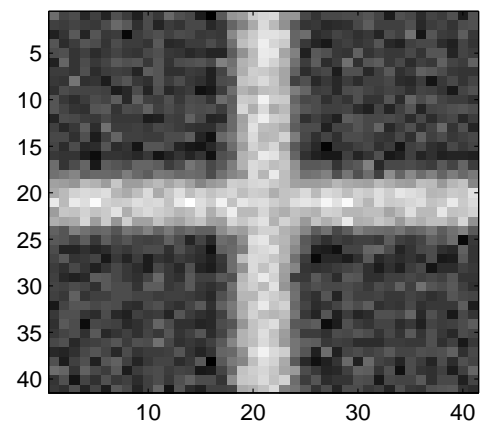

(F) OPT.FUNC.FIT (O BC)

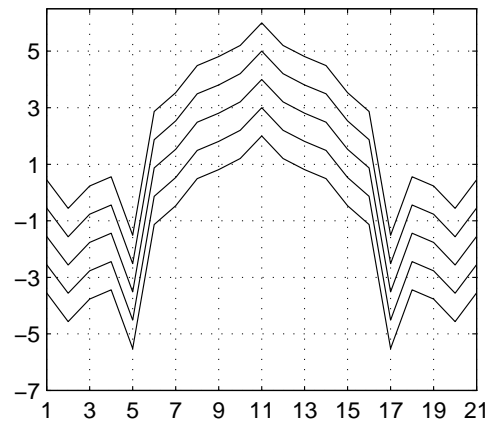

FIG. 4.3. Test 2, RSTLN and APEX/SECB results. The image was blurred using zero boundary conditions. 8-bit noise was added to obtain the image in $(B)$, resulting in $b_{\text {pert }}=1.05 \times 10^{-2}$. The blur estimate was obtained by adding 6 -bit noise to the original blur, resulting in $A_{\text {pert }}=3.91 \times 10^{-2}$. (C) STLN 2-norm solution, tol $=10^{-3}$, 26 iterations; (D) Best RSTLN 2-norm solution, $\lambda=0.75$, tol $=10^{-3}$, 25 iterations; (E) APEX/SECB recovered image using a noisy periodic image. The image was blurred as in Equation (4.2) using parameters $\alpha=0.075$ and $\beta=1$. The recovered PSF parameter estimates are $\alpha_{\text {est }}=0.0749$ and $\beta_{\text {est }}=09756$ using a scalar image component estimate of $K=2.2$. (F) APEX optimization function for a zero BC noisy image. Since the function does not have the proper form $\alpha|\xi|^{2 \beta}$, no fit can be obtained. In this case no PSF was found. 
(A) LR w/noise (per. BC)

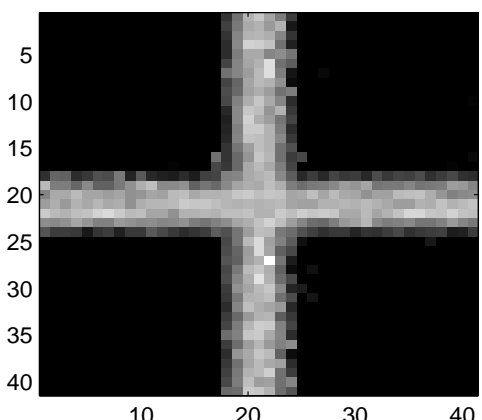

(C) M-LR w/noise (no taper, blur est.)

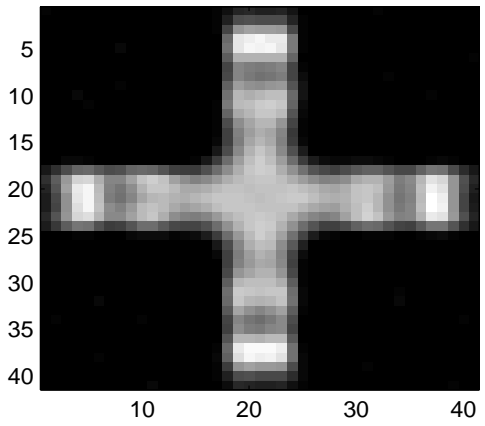

(E) M-LR w/noise (taper, blur est.)

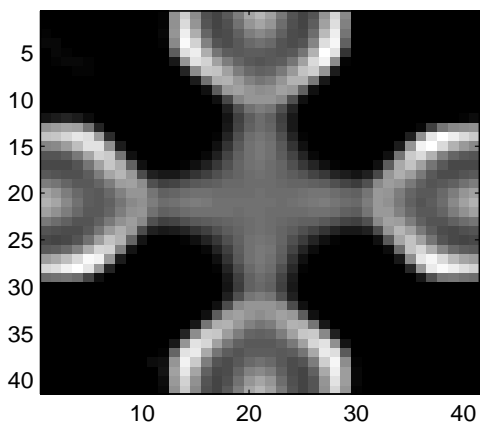

(B) LR w/noise (0 BC)

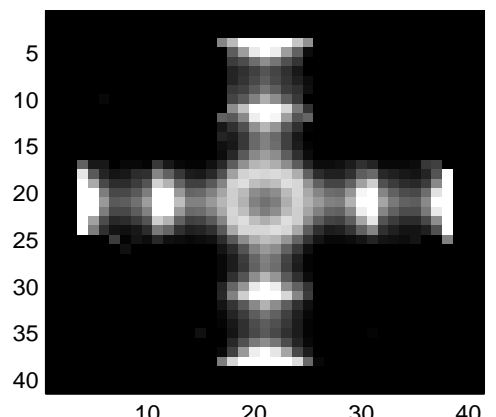

(D) M-LR w/noise (no taper, ones)

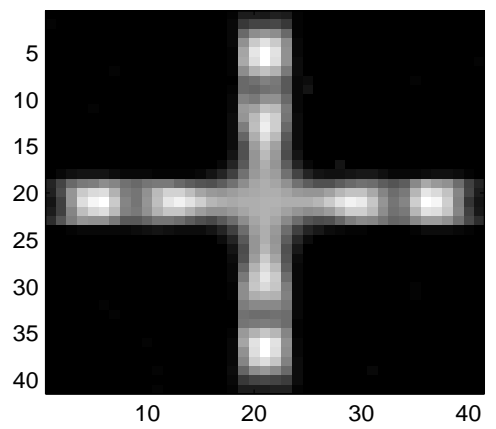

(F) M-LR w/noise (taper, ones)

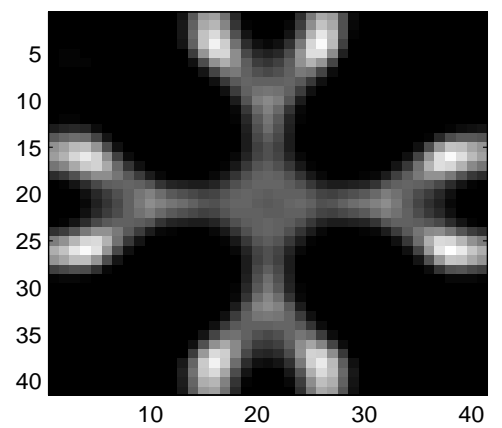

FIG. 4.4. Test 2, Lucy-Richardson results. (A) Periodic LR implementation using a periodic blurred image, 50 LR iterations each with 10 iterations; $(B)$ zero boundary LR implementation using a zero BC blurred image, 50 LR iterations each with 10 iterations; $(C) M$-LR result without tapering and using the RSTLN blur estimate, 25 iterations; (D) M-LR result without tapering and using an $11 \times 11$ matrix of ones for the PSF estimate, 25 iterations; $(E) M-L R$ result with tapering and using the RSTLN blur estimate, 10 iterations; (F) M-LR result with tapering and using an $11 \times 11$ matrix of ones for the PSF estimate, 10 iterations 
(A) ORIGINAL

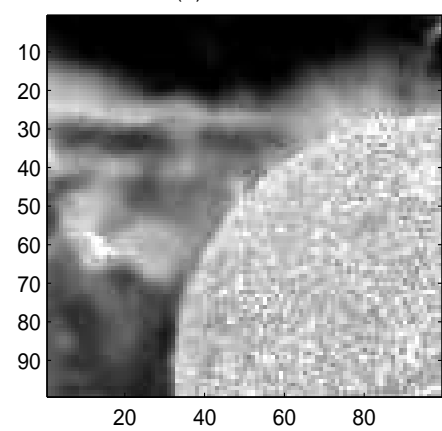

(C) STLN-2

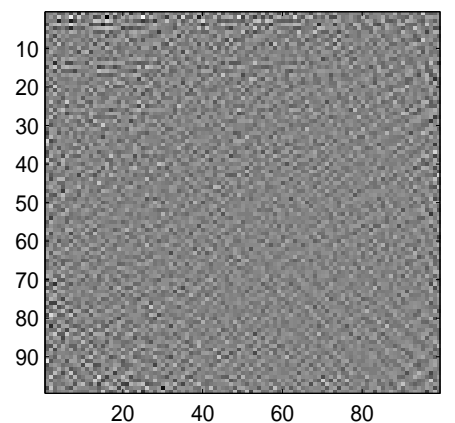

(E) APEX/SECB (PER. BC)

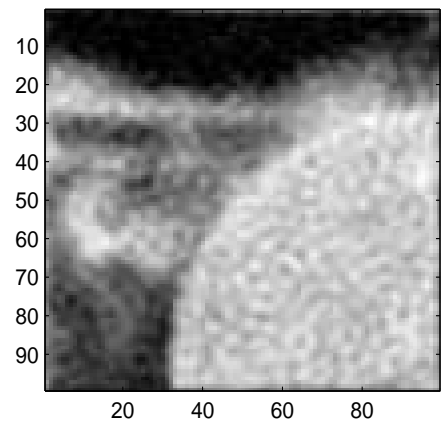

(B) NOISY BLURRED IMAGE (0 BC)

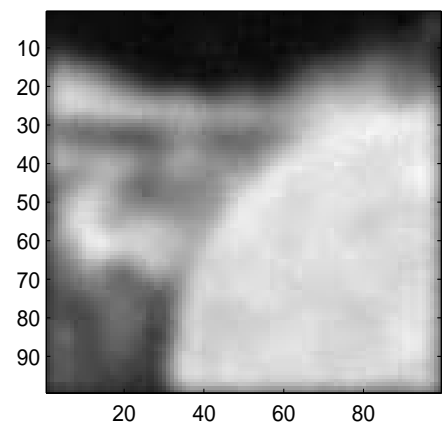

(D) RSTLN-2

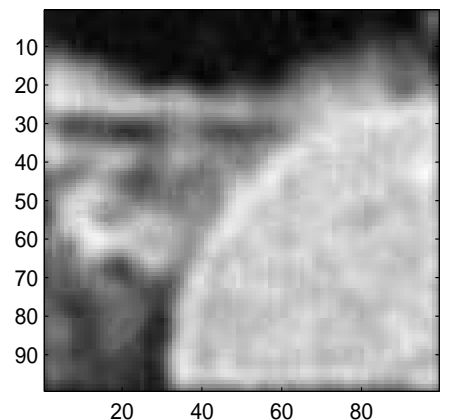

(F) OPT.FUNC.FIT (O BC)

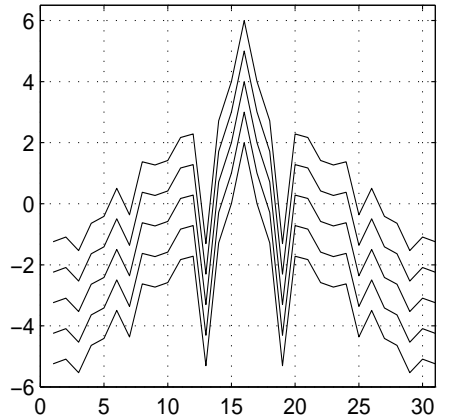

FIG. 4.5. Test 3, RSTLN and APEX/SECB results. (A) Original image $-99 \times 99$; (B) Noisy blurred image (zero BC); (C) STLN (2-norm) solution with tol $=10^{-2}$, 2 iterations; (D) RSTLN (2-norm) recovered image with initial tol $=10^{-2}$ and regularization $\lambda=75$. The experiment was stopped prematurely after 10 iterations. While larger number of iterations did achieve the desired tolerance, the results were distorted by ringing; (E) APEX/SECB recovered image. Image is blurred assuming a periodic image as in Equation 4.2 with parameters $\alpha=0.01$ and $\beta=1$. (F) Plot of optimization function if the image is blurred using zero BC. The different plots represents the optimization function for different scalar estimates for the unknown quantity $\log |\hat{f}(\xi, 0)|$, where $\hat{f}(\xi, \eta)$ denotes the normalized FFT of the original image $f$. Since none of the curves possess the proper shape, no useful PSF can be found. 
(A) LR w/noise (PER. BC)

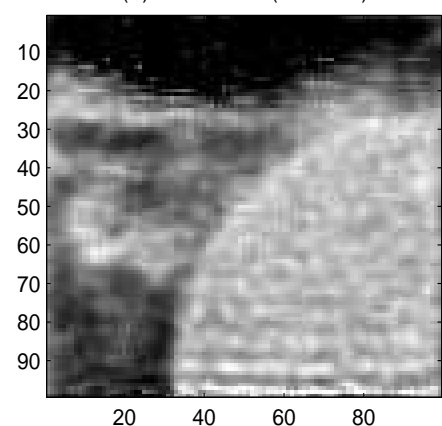

(C) M-LR w/noise (no taper, blur est.)

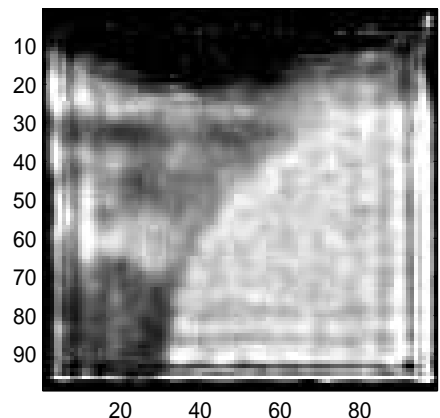

(E) M-LR w/noise (taper, blur est.)

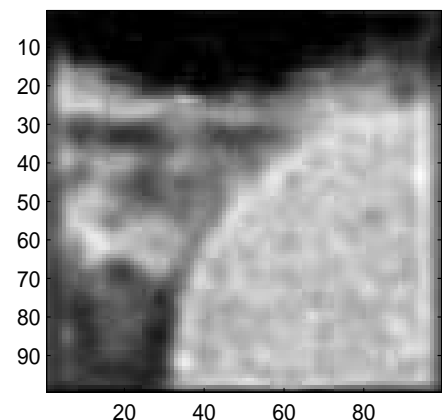

(B) LR w/noise (0 BC)

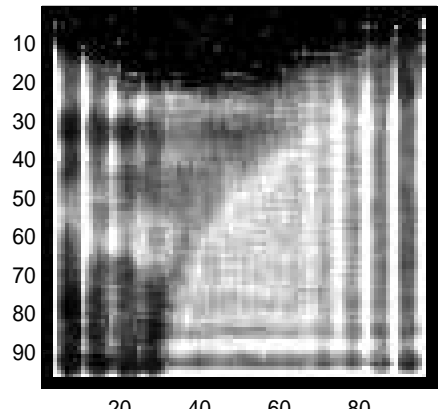

(D) M-LR w/noise (no taper, ones)

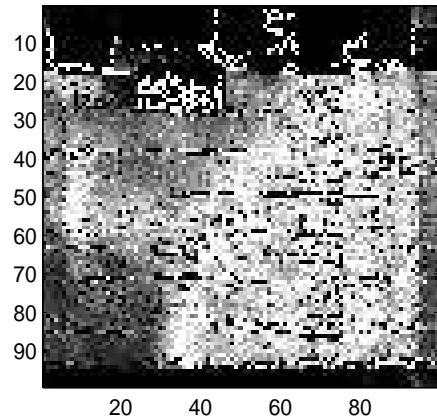

(F) M-LR w/noise (taper, ones)

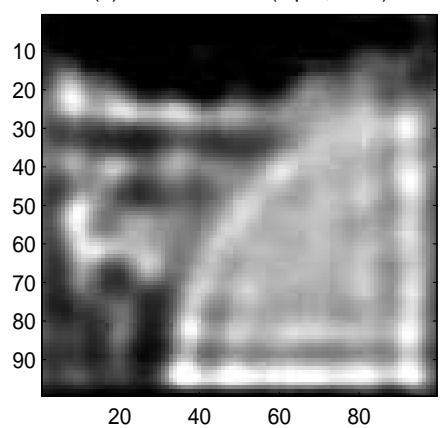

FIG. 4.6. Test 3, Lucy-Richardson results. (A) Periodic LR implementation using a periodic blurred image, 10 LR iterations each with 10 iterations; $(B)$ zero boundary LR implementation using a zero $B C$ blurred image, 15 LR iterations each with 10 iterations; $(C) M$-LR result without tapering and using the RSTLN blur estimate, 25 iterations; (D) M-LR result without tapering and using an $11 \times 11$ matrix of ones for the PSF estimate, 10 iterations; $(E) M-L R$ result with tapering and using the RSTLN blur estimate, 25 iterations; $(F)$ M-LR result with tapering and using an $11 \times 11$ matrix of ones for the PSF estimate, 10 iterations 